\title{
Esophageal cancer
}

\section{Background}

Esophageal cancer is a condition whereby malignant cancer cells arise from the inner lining of the esophagus. The two most common forms of esophageal cancer are known as squamous cell carcinoma and adenocarcinoma. Squamous cell carcinoma more commonly arises in the upper part and middle parts of the esophagus and is particularly associated with smoking and chronic alcohol consumption. Adenocarcinoma more commonly arises from the lower part of the esophagus near its junction with the stomach. Important risk factors include obesity, gastro-esophageal reflux disorder and a condition known as Barrett's esophagus, whereby gastric reflux irritates the lower esophagus and transforms normal cells into those with malignant potential. Early detection and treatment of esophageal cancer are crucial to improving one's survival and quality of life.

\section{Signs and symptoms}

Affected individuals may experience difficult or painful swallowing, first for solid foods and then for fluids as the cancer expands into an obstructive mass within the esophagus. Other signs and symptoms include progressive weight loss, nausea, vomiting, loss of appetite, chest pain and hoarseness.

\section{Diagnosis}

Initial investigations may involve imaging studies such as a barium swallow study. This involves ingestion of a liquid containing barium which lines the oesophagus and a subsequent series of X-ray images are taken so that the hollow passage within the esophagus can be visualized. For a definitive diagnosis, an endoscopy and biopsy procedure is carried out. This involves insertion of a thin tubular instrument with a camera attached into the esophagus so that tissue can be removed and examined in the lab for the presence of cancer. Once diagnosed, further imaging tests and procedures are performed to determine whether the cancer has spread to other parts of the body. These include, CT (computerized tomography) and PET (position emission tomography) scans as well as surgical procedures which use thin tube-like instruments to visualize and sample tissue from within the chest or abdomen.

\section{Management}

Treatment modalities include surgery as well as radiation therapy, chemotherapy and laser therapy. If the tumor is not amenable to surgery and is obstructing the esophagus, a metal tube, or stent, may be inserted to relieve the obstruction. Surgery, known as esophagectomy, involves removal of the part of the esophagus containing cancer and is often carried out as open surgery through incisions in the abdomen, chest or neck. For some patients with an early stage of esophageal cancer, esophagectomy may also be performed through a minimally invasive approach involving smaller incisions and the use of thin surgical instruments and a camera to visualize the operative field. For some very early stage tumors, surgical removal of the tumor may be achieved by endoscopy. The benefits of esophagectomy for appropriately selected individuals include prolonged survival, alleviation of symptoms and an improved quality of life. Important risks to consider include excessive bleeding, infection and the development of blood clots, which may in rare instances result in death. There is also a risk of leakage of gastrointestinal contents from the new surgical connection, requiring another operation. As always, the risks should be weighed against the benefits and discussed with your surgeon.

For more information, please visit the following websites: http://www.mayoclinic.org/diseases-conditions/esophagealcancer/basics/definition/con-20034316 http://my.clevelandclinic.org/health/articles/esophagealcancer http://emedicine.medscape.com/article/277930-overview

doi: $10.21037 /$ acs.2017.03.01

Section Writer: Christopher Harris

Illustration Editor: Beth Croce

Section Editor: Stine Munkholm-Larsen
For specific information concerning your medical condition, ACS suggests that you consult your physician. This page may be photocopied non-commercially by physicians to share with patients. 